\title{
Single Plasma Concentrations of 1'-Hydroxymidazolam or the Ratio of 1'-Hydroxymidazolam:Midazolam Do Not Predict Midazolam Clearance in Healthy Subjects
}

\author{
Janyce F. Rogers, PharmD, Anne N. Nafziger, MD, MHS, Angela D. M. Kashuba, PharmD, \\ Daniel S. Streetman, PharmD, Mario L. Rocci Jr., PhD, Edna F. Choo, PhD, \\ Grant R. Wilkinson, PhD, and Joseph S. Bertino Jr., PharmD
}

The 30-minute ratio of 1'-hydroxymidazolam:midazolam plasma concentrations has been used as a measure of midazolam clearance in liver transplant patients. This study determined if a single concentration of 1'-hydroxymidazolam or the ratio of 1'-hydroxymidazolam:midazolam could be used to predict midazolam clearance in healthy subjects. Plasma midazolam and 1'-hydroxymidazolam concentrations from three previous studies were used for analyses. Data obtained predose and at 5, 30, 60, 120, 240, 300, and 360 minutes following intravenous doses of midazolam in 61 adults were divided and used to derive and validate equations to predict midazolam clearance. Equations were derived using linear regression and then validated by comparing predicted to observed clearance. Only one equation was related to midazolam clearance as a function of 1'-hydroxymidazolam, but it did not predict midazolam clearance $(\mathrm{r}=0.29, \mathrm{p}=$ 0.31). Single sampling of 1'-hydroxymidazolam or 1'-hydroxymidazolam:midazolam plasma concentrations cannot be used to predict midazolam clearance in healthy adults.

Journal of Clinical Pharmacology, 2002;42:1079-1082 C2002 the American College of Clinical Pharmacology
C ytochrome P450 (CYP) phenotyping describes actual drug-metabolizing enzyme activity at any point in time. ${ }^{1}$ Phenotyping studies are used to estimate enzyme activity by correlating biomarker clearance to the amount of enzyme present. ${ }^{2}$ Intravenous (IV) midazolam is used as a hepatic cytochrome $\mathrm{P} 450$ 3A (CYP3A) phenotyping biomarker. ${ }^{2,3}$ Conven-

From the Clinical Pharmacology Research Center (Dr. Rogers, Dr. Nafziger, Dr. Bertino), Department of Medicine (Dr. Nafziger, Dr. Bertino), and Department of Pharmacy Services (Dr. Bertino), Bassett Healthcare, Cooperstown, New York; University of North Carolina, Chapel Hill (Dr. Kashuba); University of Michigan, Ann Arbor (Dr. Streetman); Pharmaceutical and Chemical Analysis, Oneida Research Services, Inc., Whitesboro, New York (Dr. Rocci); and Vanderbilt University, Nashville, Tennessee (Dr. Choo, Dr. Wilkinson). Submitted for publication January 23, 2002; revised version accepted May 26, 2002. Address for reprints: Joseph S. Bertino Jr., PharmD, Clinical Pharmacology Research Center, Bassett Healthcare, One Atwell Road, Cooperstown, NY 13326-1394.

DOI: $10.1177 / 009127002237986$

J Clin Pharmacol 2002;42:1079-1082 tionally, CYP3A activity is characterized by collecting multiple midazolam plasma concentrations over a 6- to 8-hour period to determine midazolam total systemic clearance (CL). ${ }^{2,4}$ Multiple plasma samples are costly and inconvenient, and therefore the use of minimized sampling has been investigated. Minimized sampling of plasma midazolam has been shown to be highly predictive $\left(r^{2}=0.95\right)$ of midazolam AUC using three samples. ${ }^{5}$ However, the use of a single plasma sample would be optimal. It would minimize assay costs and subject and staff time involvement. Data in liver transplant patients suggest that the plasma 1'-hydroxymidazolam:midazolam concentration ratio 30 minutes following administration of an intravenous bolus dose of midazolam is predictive $\left(r^{2}=0.76\right)$ of midazolam CL. ${ }^{6}$

The purpose of this study was to determine if a single 1'-hydroxymidazolam sample or 1'-hydroxy- 
midazolam:midazolam plasma sample ratio predicts midazolam CL in healthy adults.

\section{MATERIALS AND METHODS}

Data from three previous studies, collected at two institutions (Bassett Healthcare and Vanderbilt University), were compiled and used for these analyses. Details of the original studies have previously been published. ${ }^{1,4,7}$ A total of 61 healthy adults received an IV bolus of midazolam. The doses ranged from 1.0 to $3.1 \mathrm{mg}$. Subjects at Bassett Healthcare received midazolam 0.025 $\mathrm{mg} / \mathrm{kg}$, while subjects at Vanderbilt University received a $1 \mathrm{mg}$ dose regardless of body weight.

\section{Subjects}

Bassett Healthcare. Data from 33 healthy adults were used for these analyses. In brief, subjects underwent a medical history and physical exam prior to study participation. Subjects were nonsmokers and consumed no more than one 12-ounce beverage of beer or alcoholic equivalent per day. Subjects received IV midazolam $0.025 \mathrm{mg} / \mathrm{kg}$ (Versed $^{\circledR}: 2 \mathrm{mg} / \mathrm{ml}$ for injection, Hoffman-LaRoche, Nutley, NJ) infused over 60 seconds via an antecubital vein. Then, $7 \mathrm{ml}$ blood samples were collected into EDTA-containing tubes predose and at 5, 30, 60, 120, 240, 300, and 360 minutes following midazolam administration via an IV catheter placed in the contralateral arm. Blood samples were centrifuged. The plasma was frozen at $-80^{\circ} \mathrm{C}$ until analysis.

Vanderbilt University. Data from 28 healthy volunteers were used for the analyses. Subjects were nonsmokers and were medication free for the duration of the study. Subjects had medical histories, physical examinations, and blood work analyses prior to study participation. Subjects were given $1 \mathrm{mg}$ of IV $\left[{ }^{15} \mathrm{~N}_{3}\right]$-labeled midazolam infused over 15 to $30 \mathrm{sec}-$ onds. Blood samples were obtained predose and at 5 , $10,15,30,60,90,120,180,240,300,360$, and 480 minutes following IV midazolam administration. Collected samples were centrifuged. Plasma was frozen at $-20^{\circ} \mathrm{C}$ until analysis.

\section{Analytical Procedure}

Plasma samples were analyzed for midazolam and 1'-hydroxymidazolam concentrations using liquid chromatography/tandem mass spectrometry (LC/MS/MS). Analyses of the plasma samples collected at Bassett Healthcare were performed at Oneida
Research Services, Inc. (Whitesboro, NY). Details of these procedures have previously been published. ${ }^{4,8}$ Interassay accuracy of the LC/MS/MS assay ranged from $5.73 \%$ to $9.20 \%$ of nominal values. Interassay precision of the method was $\leq 9.89 \%$ at quality control sample concentrations of $0.75,7.5$, and $75.0 \mathrm{ng} / \mathrm{ml}$. Analyses of these plasma samples did not include a $\beta$-glucuronidase incubation period. As a result, only unconjugated plasma 1'-hydroxymidazolam was quantified.

Samples collected at Vanderbilt University were assayed on site. The LC/MS/MS assay interday reproducibility was $<12 \%$. Analyses of the plasma samples included a $\beta$-glucuronidase incubation period, allowing for quantification of total 1'-hydroxymidazolam. ${ }^{7}$

Since the 1'-hydroxymidazolam concentrations were not comparable, the data from each institution were analyzed separately. Subsequent data groups are designated as unconjugated 1'-hydroxymidazolam and total 1'-hydroxymidazolam.

\section{Pharmacokinetic Analysis}

Midazolam $\mathrm{AUC}_{0-\infty}$ were determined using the trapezoidal rule with extrapolation to infinity of the last measured midazolam concentration. The unconjugated 1'-hydroxymidazolam data were analyzed using TOPFIT 2.0 (Gustav Fischer Verlag, Stuttgart, Germany). The total 1'-hydroxymidazolam data were analyzed using NCOMP. ${ }^{7}$ Midazolam clearance was deter-

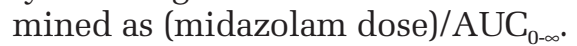

\section{Data Analysis}

Models to predict midazolam CL as a function of a single postdose concentration of 1'-hydroxymidazolam or the ratio of 1'-hydroxymidazolam:midazolam concentrations were derived using stepwise multiple linear regression (SAS 6.12, SAS Institute, Cary, NC). Sixteen of the 33 subjects from the unconjugated 1'-hydroxymidazolam group and 14 of the 28 subjects from the total 1'-hydroxymidazolam group were randomly selected and their data used to generate model equations. $p$-values $\leq 0.05$ were considered statistically significant. Regression coefficients $\left(r^{2}\right)$ for the derived equations were determined. The derived equations were then validated with data from the remaining subjects by comparing predicted CL to observed CL using the Pearson correlation coefficient $(r)$. The Wilcoxon signed rank test (SigmaStat 2.03, SPSS, Inc., Chicago) was used to determine statistically significant differ- 
Table I Demographic Information and Midazolam Clearance by Data Analysis Group for 61 Healthy Adults

\begin{tabular}{|c|c|c|c|c|c|}
\hline & \multicolumn{2}{|c|}{ Unconjugated 1'-Hydroxymidazolam Group } & \multicolumn{2}{|c|}{ Total 1'-Hydroxymidazolam Group } & \multirow[b]{2}{*}{ Total } \\
\hline & Derivation & Validation & Derivation & Validation & \\
\hline Total subjects & 16 & 17 & 14 & 14 & 61 \\
\hline Male & 7 & 7 & 14 & 14 & \\
\hline Female & 9 & 10 & 0 & 0 & \\
\hline Age range (years) & $25-51$ & $22-56$ & $25-44$ & $24-43$ & \\
\hline Age $($ mean $\pm S D)$ & $39 \pm 8.6$ & $36.7 \pm 9.3$ & $33.6 \pm 6.1$ & $33.4 \pm 5.8$ & \\
\hline Weight range (kg) & $57-102.6$ & $57-124.5$ & 68.2-115.9 & $75.7-102.7$ & \\
\hline Weight (mean $\pm S D$ ) & $77.0 \pm 16.8$ & $77.8 \pm 16.7$ & $88.4 \pm 2.2$ & $85 \pm 9.1$ & \\
\hline Height range (cm) & 158-184 & 157-188 & 165-185 & 165-193 & \\
\hline Height (mean $\pm S D$ ) & $169.4 \pm 7.7$ & $170.5 \pm 8.9$ & $178.0 \pm 7.2$ & $177.5 \pm 7.5$ & \\
\hline \multicolumn{6}{|l|}{ Race, $n(\%)$} \\
\hline Caucasian & 16(100) & 17(100) & $7(50)$ & $8(57)$ & $48(79)$ \\
\hline African American & $0(0)$ & $0(0)$ & $7(50)$ & $6(43)$ & $13(21)$ \\
\hline \multicolumn{6}{|l|}{ Midazolam clearance } \\
\hline Range (ml/min) & 404-718 & $437-836$ & $194-381$ & 198-391 & \\
\hline Mean $\pm S D$ & $539 \pm 87$ & $608 \pm 118$ & $282 \pm 65$ & $297 \pm 55$ & \\
\hline Median & 534 & 581 & 266 & 296 & \\
\hline
\end{tabular}

No statistically significant differences in demographic information $(p>0.05)$ exist between derivation and validation subsets of either group.

ences between demographic data for each group (derivation and validation).

\section{RESULTS}

Demographic data for the derivation and validation groups are shown in Table I.

Single sampling strategies were investigated at all time points (i.e., 5, 30, 60, 120, 240, 300, and 360 min) following the dose of midazolam. No model derived from the unconjugated 1'-hydroxymidazolam data, using 1'-hydroxymidazolam or 1'-hydroxymidazolam:midazolam, was predictive of midazolam CL.

No model derived from the total 1'-hydroxymidazolam data to predict midazolam CL as a function of a single time point 1'-hydroxymidazolam:midazolam was predictive of midazolam CL. One model predicted midazolam CL as a function of total 1'-hydroxymidazolam. The equation is as follows:

$$
\mathrm{CL}=445.9-65.1 \text { (total 1'-hydroxymidazolam } 300 \mathrm{~min} \text { ). }
$$

The corresponding $r^{2}=0.29$ had a $p<0.0001$. This derived equation was then validated with data from the remaining 14 participants. Pearson correlation was used to compare predicted midazolam CL to observed midazolam CL (Figure 1). The derived equation could not be validated $(r=0.29, p=0.31)$.

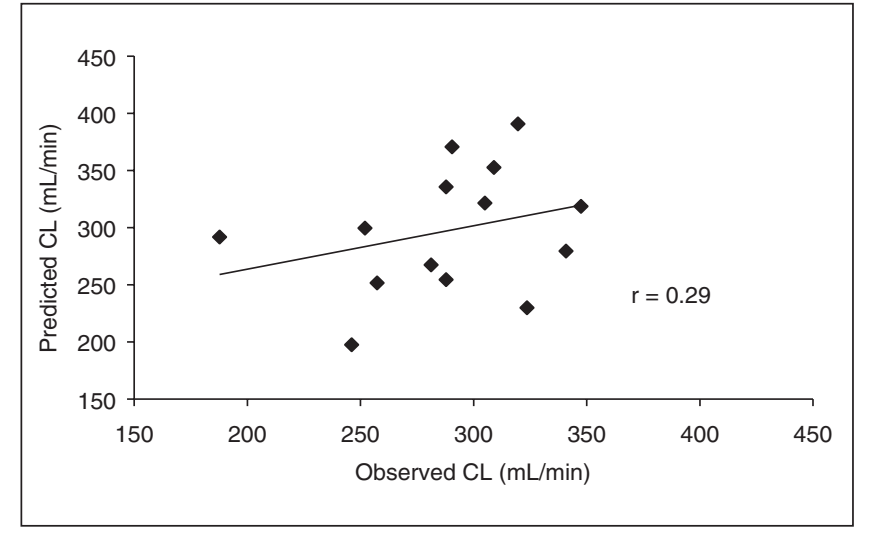

Figure 1. Observed midazolam clearance versus predicted midazolam clearance based on the 300-minute total 1'-hydroxymidazolam concentration with corresponding Pearson correlation coefficient (r).

\section{DISCUSSION}

Limited sampling has been demonstrated with the use of midazolam, ${ }^{6,9}$ and single sampling of omeprazole and 5-hydroxyomeprazole concentrations 3 hours following drug administration is often used for CYP2C19 phenotyping. ${ }^{10,11}$ While the limited sampling strategy for midazolam is more convenient than standard sampling, single sampling would be optimal. 
A previous study showed that a single sampling approach was predictive of midazolam clearance in liver transplant patients. However, this correlation was dependent on steroid-induced variability. ${ }^{6}$ As a result, the use of this 30-minute ratio would most likely have minimal utility in predicting midazolam clearance in healthy subjects. The results of our data analyses showed failure of any single sampling time point of 1'-hydroxymidazolam or the ratio of 1'-hydroxymidazolam:midazolam to predict midazolam clearance. A possible reason for this failure is intersubject variability associated with genetic and environmental factors. Also, midazolam is lipophilic, and its volume of distribution is increased in obese subjects. ${ }^{12}$ Some study participants were obese and therefore have larger volumes of distribution. In addition, midazolam is moderately extracted by the liver, where the hepatic extraction ratio $\left(\mathrm{E}_{\mathrm{H}}\right)$ is $0.3 \leq \mathrm{E}_{\mathrm{H}} \leq 0.7$. As such, the clearance of midazolam is dependent on CYP3A activity, hepatic blood flow, and the fraction of unbound midazolam in the plasma. The influence of all of these factors will add intersubject variability to resulting midazolam clearance.

Single sampling strategies using 1'-hydroxymidazolam:midazolam or 1'- hydroxymidazolam concentrations cannot be used to predict midazolam CL in healthy adults. Minimized sampling (i.e., 5, 30, 360 $\mathrm{min}$ ) of midazolam concentrations remains a more reliable method for estimating midazolam AUC. Midazolam CL can then be calculated and correlated to hepatic CYP3A activity.

\section{REFERENCES}

1. Streetman DS, Bleakley JF, Kim JS, Nafziger AN, Leeder JS, Gaedigk A, et al: Combined phenotypic assessment of CYP1A2, CYP2C19, CYP2D6, CYP3A, N-acetyltransferase-2, and xanthine oxidase with the "Cooperstown cocktail." Clin Pharmacol Ther 2000;68:375-383.
2. Watkins PB: Noninvasive tests of CYP3A enzymes. Pharmacogenetics 1994;4:171-184.

3. Streetman DS, Bertino JS Jr, Nafziger AN: Phenotyping of drugmetabolizing enzymes in adults: a review of in-vivo cytochrome P450 phenotyping probes. Pharmacogenetics 2000;10:187-216.

4. Kashuba AD, Bertino JS Jr, Rocci ML Jr, Kulawy RW, Beck DJ, Nafziger AN: Quantification of 3-month intraindividual variability and the influence of sex and menstrual cycle phase on CYP3A activity as measured by phenotyping with intravenous midazolam. Clin Pharmacol Ther 1998;64:269-277.

5. Kim JS, Nafziger AN, Tsunoda SM, Choo EF, Streetman DS, Kashuba AD, et al: Limited sampling strategy to predict AUC of the CYP3A phenotyping probe midazolam in adults: application to various assay techniques. J Clin Pharmacol 2002;42:376-382.

6. Thummel KE, Shen DD, Podoll TD, Kunze KL, Trager WF, Bacchi $\mathrm{CE}$, et al: Use of midazolam as a human cytochrome P450 3A probe: II. Characterization of inter- and intraindividual hepatic CYP3A variability after liver transplantation. J Pharmacol Exp Ther 1994; 271:557-566.

7. Wandel C, Witte JS, Hall JM, Stein CM, Wood AJ, Wilkinson GR: CYP3A activity in African American and European American men: population differences and functional effect of the CYP3A4*1B5'-promoter region polymorphism. Clin Pharmacol Ther 2000;68:82-91.

8. Fayer JL, Zannikos PN, Stevens JC, Luo Y, Sidhu R, Kirkesseli S: Lack of correlation between in vitro inhibition of CYP3A-mediated metabolism by a PPAR-gamma agonist and its effect on the clinical pharmacokinetics of midazolam, an in vivo probe of CYP3A activity. J Clin Pharmacol 2001;41:305-316.

9. Kim JS, Nafziger AN, Tsunoda SM, Choo EF, Streetman DS, Kashuba AD, et al: Validation of a minimized plasma sampling strategy (MPSS) to calculate AUC of the CYP3A probe midazolam (MID). Clin Pharmacol Ther 2000;67:161.

10. Sagar M, Tybring G, Dahl ML, Bertilsson L, Seensalu R: Effects of omeprazole on intragastric $\mathrm{pH}$ and plasma gastrin are dependent on the CYP2C19 polymorphism. Gastroenterology 2000;119:670-676.

11. Roh HK, Dahl ML, Tybring G, Yamada H, Cha YN, Bertilsson L: CYP2C19 genotype and phenotype determined by omeprazole in a Korean population. Pharmacogenetics 1996;6:547-551.

12. MICROMEDEX ${ }^{\circledR}$ Healthcare Series. MICROMEDEX, Greenwood Village, CO (edition expires 6/2002). 